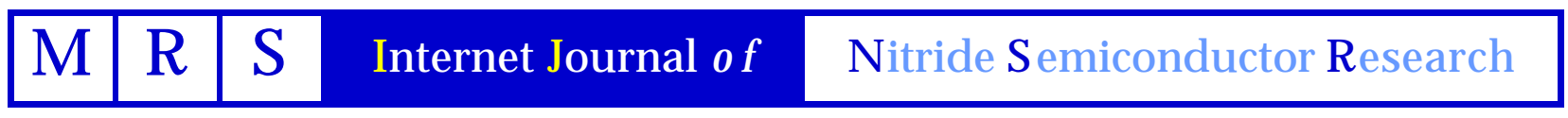

Volume 2, Article 35

\title{
Theoretical Studies Of Hydrogen Passivated Substitutional Magnesium Acceptor In Wurzite GaN
}

\author{
V. J. B. Torres \\ Departamento de Física, Universidade de Aveiro \\ S. Öberg \\ Department of Mathematics, University of Luleå, \\ R. Jones \\ Departrnent of Physics, University of Exeter
}

This article was received on July 15, 1997 and accepted on September 15, 1997.

\begin{abstract}
Infrared measurements on wurzite $\mathrm{GaN}$ codoped with $\mathrm{Mg}$ and $\mathrm{H}$ reveal strong absorption at $3125 \mathrm{~cm}^{-1}$. Theorectical work provides strong evidence for the $\mathrm{H}$ being antibonding to $\mathrm{N}$. We have performed an ab-initio study of $\mathrm{Mg}-\mathrm{H}$ complexes in wurzite GaN, using the Local Density Approximation on a large $\mathrm{H}$-terminated cluster $\mathrm{MgHGa}_{25} \mathrm{~N}_{26} \mathrm{H}_{42}$. We have investigated the physical properties of three neutral configurations along the $\mathrm{c}$ direction. In all configurations $\mathrm{Mg}$ sits in a gallium substitutional site. $\mathrm{H}$ is then located in the Mg-N bond centre $(B C)$, in the antibonding site on nitrogen side $\left(A B_{N}\right)$ or in the antibonding position on magnesium side $\left(\mathrm{AB}_{\mathrm{Mg}}\right)$. We found the lowest total energy configuration is hydrogen in the antibonding on the nitrogen site. The stretch mode in this configuration is calculated at $3277 \mathrm{~cm}^{-1}$ which agrees with experiment and previous LDA calculations and we predict an unreported infra red active wag mode at $1311 \mathrm{~cm}^{-1}$. The experimental isotopic shift with $D$ is well reproduced. The $B C$ and $A_{\mathrm{Mg}}$ configurations are 0.5 and $3.7 \mathrm{eV}$ higher, producing local modes at 3645 and $2144 \mathrm{~cm}^{-1}$, respectively. No wag modes appear for the $B C$ and $A B_{M g}$ configurations.
\end{abstract}

\section{Introduction}

Hydrogen is often present in semiconductors and may passivate the acceptor or donor electronic level [1]+ [2]+ [3], decreasing the carrier concentration. Hydrogen passivation has been observed in silicon, germanium, III-V and II-VI materials. In wurzite GaN, hydrogen passivates magnesium [4] and calcium [5] acceptors.

Early infra red absorption experiments [6] on $\mathrm{Mg}$ doped wurzite $\mathrm{GaN}$, with $\mathrm{H}$ incorporated unintentinally, showed two lines at 2168 and $2219 \mathrm{~cm}^{-1}$, which were attributed to a $\mathrm{Mg}-\mathrm{H}$ centre. However ab-initio calculations obtained much higher local vibrational modes (LVM), between 2930 [7] and $3360 \mathrm{~cm}^{-1}$ [8] for the lowest formation energy configuration, $\mathrm{H}$ antibonding to nitrogen. This disagreement prompted further infrared experiments. New IR experiment [9], sensitive to vibrations on c plane, which detected a new local vibrational mode at $3125 \mathrm{~cm}^{-1}$. For deuterated samples a LVM at $2321 \mathrm{~cm}^{-1}$ emerged. The ratio of the last two frequencies to be near $\sqrt{2}$ shows that hydrogen is involved in the complex. This new experimental data is in good concordance with predicted theoretical quantities, validating the antibonding to nitrogen model.

Ab-initio calculations by Neugebauer and Van de Walle [8] and by Bosin, Fiorentini and Vanderbildt [7] have shown that Mg-H LVMs are similar for cubic and for wurzite GaN in the c direction and in the $c$ plane. In this paper we present a study of the Mg-H complex in c direction in wurzite GaN. We compare the total energy and the LVMs of three possible neutral configurations with $\mathrm{C}_{3 \mathrm{v}}$ symmetry. All configurations consider $\mathrm{Mg}$ at a gallium 
substitutional site and $\mathrm{H}$ sitting in the $\mathrm{Mg}-\mathrm{N}$ bond centre $(\mathrm{BC})$, in the antibonding site on nitrogen side $\left(A B_{N}\right)$ and in the antibonding position on magnesium side $\left(\mathrm{AB}_{\mathrm{Mg}}\right)$. Calculations of hydrogen passivation of others acceptors (Be, $\mathrm{Ca}, \mathrm{Zn}$ and $\mathrm{Cd}$ ) in wurzite $\mathrm{GaN}$ are in progress and will be presented in a separate publication.

\section{Method}

We use an ab initio local density functional method on a large $\mathrm{H}$-terminated cluster [10]. Norm conserving pseudopotentials [11] were used. The electronic wavefunction and the charge density were expanded in s- and p-type gaussians atomic orbitals, centred on nuclei and at the centre of all non $\mathrm{H}$-terminated bonds.

The total self-consistent energy is found and the atomic forces calculated analitically. A conjugated gradient algorithm is used to minimize the total energy. In our calculations we use a $\mathrm{MgHGa}_{25} \mathrm{~N}_{26} \mathrm{H}_{42}$ atomic cluster, centred on the middle of a Ga-N bond, and all atoms were allowed to relax. We verified the convergence of the results presented here with respect to basis set.

Second derivatives of the energy in respect to the atomic positions are obtained numerically and inserted into the dynamical matrix to calculate the LVMs. In pratice, the numerically calculated second derivatives were determined for the atoms involved in the defect and their first nearest neighbours. Outside the defect region, the second derivatives are calculated using a Keating Potential [12]. We consider non-Coulombic bond stretching and bond bending interactions. The parametrization was chosen to fit the highest frequency at the $\Gamma$ point and the bulk modulus for cubic GaN.

\section{Results}

We have determined the relaxed atomic geometry, total energy and LVMs for three neutral configurations, BC, $A B_{N}$ and $A B_{M g}$, along the $c$ direction.

We found $A B_{N}$ to be the lowest total energy configuration, while $B C$ and $A B_{M g}$ configurations are 0.5 and $3.7 \mathrm{eV}$ higher, respectively.

For the $A B_{N}$ configuration nitrogen moved $0.12 \AA$ towards the $M g$ site, while magnesium moved $0.03 \AA$ away from $\mathrm{N}$, resulting in a Mg-N bond length of $1.89 \AA$, close to the $\mathrm{Ga}-\mathrm{N}$ ideal bond length. The $\mathrm{N}-\mathrm{H}$ bond length is $1.02 \AA$. In the $\mathrm{BC}$ configuration nitrogen and magnesium moved apart 0.03 and $0.35 \AA$, respectively. The $\mathrm{N}-\mathrm{H}$ bond length is $0.99 \AA$. The $\mathrm{Mg}-\mathrm{N}$ distance is $0.4 \AA$ greater than the perfect bond length, which increases the strain energy.

The local modes are listed in Table 1. The lowest total energy configuration, $A B_{N}$, produces 3327 and $2387 \mathrm{~cm}$

-1 stretch modes for hydrogen and for deuterium respectively, in good agreement with experiment and previous calculations. Replacing ${ }^{14} \mathrm{~N}$ by ${ }^{15} \mathrm{~N}$ low the $3327 \mathrm{~cm}^{-1}$ stretch mode by $6 \mathrm{~cm}^{-1}$. We also predict an unreported wag mode at $1311 \mathrm{~cm}^{-1}$. The $B C$ and the $A_{B g}$ configurations produce stretch local modes at 3645 and $2144 \mathrm{~cm}^{-1}$, for hydrogen, and 1552 and $2650 \mathrm{~cm}^{-1}$ for deuterium, respectively. These LVMs do not agree with experiment. No wag local modes were found for these two configurations. To verify the local modes dependence on the Keating Potential parametrization, the Keating force contants were varied by $30 \%$. No significant changes on local modes were found.

\section{Conclusions}

We find that the lowest total energy configuration for the $\mathrm{Mg}-\mathrm{H}$ complex along the c direction in wurzite $\mathrm{GaN}$ hydrogen sits at anti-bonding site at the nitrogen nearest neighbour of $\mathrm{Mg}_{\mathrm{Ga}}$. This configuration produces a stretch local mode in good agreement with experiment and previous calculations by Neugebauer and Van de Walle and by Bosin, Fiorentini and Vanderbilt. The experimental isotopic shift for deuterium is well reproduced and is predited to be $6 \mathrm{~cm}^{-1}$ lower for ${ }^{15} \mathrm{~N}-\mathrm{H}$ We also found an unreported infra red active wag local mode for the $A B_{N}$ configuration at $1311 \mathrm{~cm}^{-1}$. The $B C$ and $A B_{M g}$ configurations do not show any wag local modes.

\section{Acknowledgments}


V. Torres acknowledges the financial support of JNICT, contract PRAXIS XXI PBIC/C/CTM/1925/95.

\section{References}

[1] C. T. Sah, J. Y. -C. Sun, J. J. Tzou, Appl. Phys. Lett. 43, 204 (1983).

[2] J. I. Pankove, D. E. Carlson, J. E. Berkeyheiser, R. O. Wance, Phys. Rev. Lett. 51, 2224 (1983).

[3] N. M. Johnson, Phys. Rev. B 69, 3725 (1985).

[4] M. S. Brandt, N. M. Johnson , R. J. Molnar, R. Singh, T. D. Moustakas , Appl. Phys. Lett. 64, 2264-2266 (1994).

[5] J. W. Lee, S. J. Pearton , J. C. Zolper , R. A. Stall , Appl. Phys. Lett. 68, 2102-2104 (1996).

[6] M. S. Brandt , J. W. Ager, III, W. Gotz, N. M. Johnson , J. S. Harris, Jr., R. J. Molnar, T. D. Moustakas , Phys. Rev. B 49, 14758-14761 (1994).

[7] A. Bosin, V. Fiorentini, D. Vanderbilt, Mater. Res. Soc. Symp. Proc. 395, 503 (1996).

[8] Jorg Neugebauer, Chris G. Van de Walle, Phys. Rev. Lett. 75, 4452-4455 (1995).

[9] W. Gotz, N. M. Johnson, D. P. Bour, M. D. McCluskey, E. E. Haller, Appl. Phys. Lett. 69, 3725 (1996).

[10]R. Jones, "Ab initio cluster calculations of defects in solids", Phil. Trans. Roy. Soc. London Ser. A, 341, 351 (1992)

[11] G. B. Bachelet, D. R. Hamann, M. Schlüter, Phys. Rev. B 26, 4199-4228 (1982).

[12] P. N. Keating, Phys. Rev. 145, 637 (1966).

\section{Table 1}

Calculated and Experimental LVMs of Mg-H (Mg-D) complex in wurzite GaN $\left(\mathrm{cm}^{-1}\right)$.

\begin{tabular}{|c|c|c|c|c|c|}
\hline \multirow[t]{3}{*}{ Configurations } & \multirow{2}{*}{\multicolumn{2}{|c|}{$\begin{array}{c}\text { Present } \\
\text { (c direction) }\end{array}$}} & \multirow{3}{*}{\begin{tabular}{|c|} 
Ref. [8] \\
(cubic GaN) \\
Stretch \\
\end{tabular}} & \multicolumn{2}{|c|}{ Ref. [7] } \\
\hline & & & & \multirow{2}{*}{$\begin{array}{c}\text { (c direction) } \\
\text { Stretch }\end{array}$} & \multirow{2}{*}{\begin{tabular}{|l} 
(c plane) \\
Stretch
\end{tabular}} \\
\hline & Stretch & Wag & & & \\
\hline \multirow{2}{*}{$\mathrm{AB}_{\mathrm{Mg}}$} & 2144 & & & & \\
\hline & $(1552)$ & & & & \\
\hline \multirow{2}{*}{$\mathrm{BC}$} & 3645 & & & 3611 & 3917 \\
\hline & $(2650)$ & & & & \\
\hline \multirow{2}{*}{$\mathrm{AB}_{\mathrm{N}}$} & 3277 & 1311 & 3360 & 3069 & 2930 \\
\hline & (2387) & $(1023)$ & & & \\
\hline \multirow{2}{*}{$\begin{array}{c}\text { Expt. } \\
\text { (c plane) }\end{array}$} & 3125 & & & & \\
\hline & $(2321)$ & & & & \\
\hline
\end{tabular}

(C) 1997 The Materials Research Society

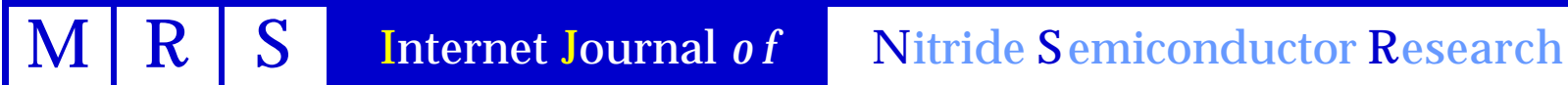

